\title{
Es ist Frühling - und wie!
}

N

ach einem langen Winter hat der geballt einsetzende Pollenflug in diesem Jahr die allergologischen Praxen mit zum Teil schwer kranken Patienten gefüllt. Auffällig ist die Häufigkeit von schwer kranken Pollenasthmatikern. Selbst Patienten, die in den letzten Jahren nur wenige oder überhaupt keine Beschwerden hatten, weisen jetzt massive asthmatische Symptome auf. Auf der Internet-Seite der Stiftung Deutscher Pollenfluginformationsdienst (www.pollenstiftung.de) können entsprechende Daten zum ausgeprägten Birkenpollenflug gefunden werden.

Was aber können wir diesen Patienten bieten? Da wir die meisten Antihistaminika nur dann zu Lasten der gesetzlichen Krankenversicherung verordnen dürfen, wenn bereits topische Glukokortikoide zum Einsatz gekom-
„Wir sollten uns auf die Kernkompetenz der Allergologen konzentrieren: gezielte Beratung und die frühzeitige Einleitung der spezifischen Immuntherapie."

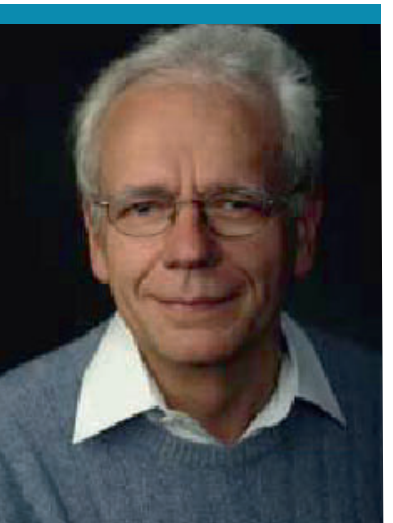

Dr. Wolfgang Rebien, Hamburg, Präsident des ÄDA

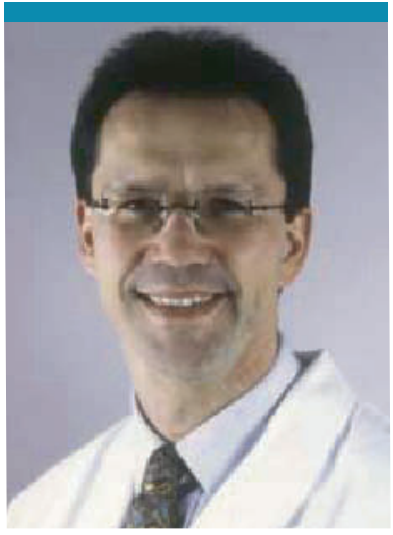

Prof. Dr. Ludger Klimek, Zentrum für Rhinologie und Allergologie, Wiesbaden, Vorstandsmitglied des ÄDA handlung dieser zum Teil schwer verlaufenden Erkrankung erleichtert.

Ferner finden wir in dieser Ausgabe eine interessante Kasuistik von Daniela Klode, bei der eine Patientin auf das Schmerzmittel Prophenazon im Sinne einer Soforttypallergie reagiert hat.

Abschließend dürfen wir für den September dieses Jahres in die aufwendig restaurierten Jugendstil-Räumlichkeiten des Kurhauses in Wiesbaden zum 29. Kongress des Ärzteverbandes Deutscher Allergologen einladen. Diese Tagung wird traditionell als interdisziplinäre Fortbildungsveranstaltung für alle

\section{„Abschließend dürfen wir Sie für den September dieses Jahres nach Wiesbaden zum 29. Kongress des Ärztever- bandes Deutscher Allergo- logen einladen."} Standardisierung von oralen Provokationstests bei Nahrungsmittelallergien" unter der Leitung des auf diesem Gebiet besonders erfahrenen Bodo Niggemann erarbeitet. Für den auf dem Gebiet der Nahrungsmittelallergie arbeitenden Allergologen liegt hier eine bis ins Detail durchdachte Anleitung zur Durchführung der oralen Nahrungsmittelprovokation vor.

In einer Übersichtsarbeit von Kurt Brockow werden Marker zusammengestellt, mit denen Rückschlüsse auf den Schweregrad einer Mastozytose gezogen werden können (Mastzelltryptase, Histamin, IL-6 und lösliches CD25 und CD117). Hierdurch werden sowohl die Beratung der Patienten als auch die Beallergologisch tätigen Ärzte aus Dermatologie, HNO-Heilkunde, Pneumologie/Innerer Medizin und Pädiatrie durchgeführt.

Wir freuen uns darauf, Sie beim Allergie-Kongress 2006 in Wiesbaden am 15. und 16. September begrüßen zu dürfen!

Mit kollegialen Grüssen
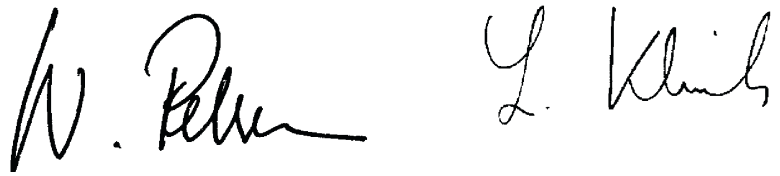

Dr. Wolfgang Rebien

Prof. Dr. Ludger Klimek 